Original Paper http://ajol.info/index.php/ijbcs $\quad$ http://indexmedicus.afro.who.int

\title{
Evaluation de lignées intra et inter spécifiques de riz en conditions irriguées au Sénégal
}

\author{
Tala GUEYE*, Saliou NDIAYE et Abdoulaye DIENG \\ Université de Thiès, Ecole Nationale Supérieure d'Agriculture (ENSA), BP A296, Sénégal. \\ "Auteur correspondant ; E-mail: tgueye@refer.sn, Tel : 00221775691595
}

\section{RESUMÉ}

Parmi les deux espèces de riz cultivées, l'espèce africaine Oryza glaberrima Steudt. est une source de gènes utiles pour des caractères importants, qui n'existent pas chez l'espèce asiatique Oryza sativa L., limitant ainsi ses performances en Afrique. Cela a inspiré le processus d'hybridation interspécifique entre ces espèces pour développer un nouveau type de riz mieux adapté aux conditions de production du paysan africain. Dans l'optique d'élargissement de la diversité génétique du riz cultivé en conditions irriguées, l'évaluation des performances de 31 lignées intra- et interspécifiques, a été effectuée, dans un dispositif à blocs de Fisher à 2 répétitions, sur deux sites et deux saisons, dans la vallée du fleuve Sénégal. L'analyse de variance combinée sur site et saison a montré un effet très significatif du génotype sur l'ensemble des caractères étudiés. Un effet significatif de la saison sur le cycle et de l'interaction Génotype x Site (G x E) sur la hauteur a aussi été observé. Cependant, aucun effet $\mathrm{G} \times \mathrm{E}$ sur le rendement et le nombre de panicules par $\mathrm{m}^{2} \mathrm{n}$ 'a été observé. Les rendements moyens varient entre 6,61 et 8,78 t.ha ${ }^{-1}$. Les lignées prometteuses identifiées devraient contribuer à l'amélioration des performances du riz cultivé dans la région.

(C) 2016 International Formulae Group. All rights reserved.

Mots clés : Sénégal, lignées, diversité génétique, rendement, riziculture irriguée.

\section{Evaluation of intra- and interspecific rice lines under irrigated conditions in Senegal}

\begin{abstract}
Among the two cultivated rice species, the African species Oryza glaberrima Steudt. is a source of useful genes for important traits, which do not exist in the Asian species Oryza sativa L., limiting thus its performance, in Africa. This has inspired the interspecific hybridization process between the two species to develop a new type of rice best suited to the production conditions of the African peasant. With the aim of broadening the genetic diversity cultivated rice in irrigated conditions, the performance evaluation of 31 lines, intra- and inter, was conducted in a randomized complete block design with 2 replications, on two sites and two seasons in the Senegal River valley. The analysis of variance combined over site and season shows a highly significant difference of Genotype $(G)$ for all studied traits. A significant effect of season on cycle, and interaction genotype $\mathrm{x}$ location $(\mathrm{G} \times \mathrm{E})$ on plant height, was also observed. However, no (G x E) effect on yield and number of panicles per quadrate meter were observed. The average of yield varies between 6.61 and 8.78
\end{abstract}


t.ha $^{-1}$. Identified promising lines should contribute to the sustainable improvement of the performance of rice cultivated in the area.

Keywords: Senegal, lines, genetic diversity, grain yield, irrigated rice.

\section{INTRODUCTION}

La riziculture irriguée dans la Vallée du Fleuve Sénégal est une agriculture intensive dont les potentialités ne sont pas encore exploitées de manière optimale. Elle présente l'avantage d'être indemne des principales maladies fongiques (Pyriculariose), bactériennes ou virales (RYMV). La disponibilité de l'eau douce du fleuve durant toute l'année, permet la pratique de la double culture, en hivernage et durant la contre saison chaude. Les paysans de la vallée peuvent donc récolter deux fois par an, et contribuer à l'augmentation de la production alimentaire, à travers l'augmentation de la fréquence des récoltes (Hu et al., 2015). Cependant, des contraintes diverses existent, dont la salinité, qui affecte $25 \%$ des superficies rizicoles en Afrique de l'Ouest (Bimpong et al. 2016), la pression des mauvaises herbes qui cause des pertes considérables dans les écosystèmes rizicoles (Haefele et al., 2004 ; Gaouna et al., 2012), la pression parasitaire (foreurs de tiges, défoliateurs et suceurs de sèves), la faiblesse de la mécanisation agricole, la non utilisation systématique de semences certifiées par une frange importante des producteurs, le non respect des itinéraires techniques, et les conditions socioéconomiques qui limitent la production rizicole dans la vallée. Les rendements moyens dans la plupart des superficies aménagées, tournent autour de 5,5 t.ha ${ }^{-1}$. Ces rendements ont été obtenus sur les variétés Sahel 108, Sahel 201 et Sahel 202, qui sont les trois principales variétés présentes dans la plupart des superficies aménagées dans la région. Elles sont issues de la collection de l'IRRI et ont été inscrites au catalogue variétal du Sénégal depuis 1994, suite à différents tests d'évaluation. Depuis, elles ont progressivement remplacé la plupart des anciennes variétés introduites, comme IR8, D 52-37, KSS, IKP et JAYA, devenues de plus en plus rares voire inexistantes pour certaines d'entre elles. Cinq (5) autres variétés dont deux à cycles courts et trois à cycles moyens, récemment homologuées, sont en phase de diffusion. Ces différentes variétés ont en commun leur origine asiatique, et ont une grande sensibilité aux conditions adverses des écosystèmes africains. La recherche de variétés plus résistantes et mieux adaptées aux conditions socioéconomiques locales et aux contraintes du milieu, est devenue, depuis une dizaine d'années, un objectif majeur, dans un contexte de relance de la production nationale. Cela passe nécessairement par l'élargissement de la diversité génétique des variétés cultivées pour les rendre plus aptes aux contraintes du milieu. Le développement d'hybrides interspécifique qui combinent les meilleures caractéristiques du riz cultivé africain, Oryza glaberrima Steudt., plus adapté aux conditions du milieu, et du riz asiatique, $O$. sativa $\mathrm{L}$., à rendement plus élevé, obéit à cette stratégie (Jones et al., 1997 ; Ndjiondjop et al., 2010 ; Yacouba et al., 2013). Les tests de lignées recombinées sont importants dans le processus de création de nouvelles variétés (Banito et al., 2012 ; Goalbaye et al., 2013, Bustos et al., 2013 ; Li et al., 2015). Comme pour les autres écosystèmes de culture du riz, le Génotype et l'Environnement de production sont les facteurs clés qui impactent les performance en grain (Liang et al., 2015). Suite à un programme de croisement, des lignées recombinées fertiles avaient été obtenues après plusieurs générations de rétro croisements. L'objectif de cette étude est de tester le comportement des lignées intra- et interspécifiques, afin d'identifier des génotypes prometteurs, adaptées à la riziculture irriguée.

\section{MATERIEL ET METHODES}

Le matériel végétal est constitué de trente et une (31) lignées inter- et intra spécifiques et quatre (4) témoins, constitués 
de variétés les plus cultivées dans la région.

Les entrées ont été semées en lignes dans un dispositif en bloc de Fisher à 2 répétitions, avec un écartement intra et interligne de $20 \mathrm{~cm}$ dans des parcelles élémentaires de $12 \mathrm{~m}^{2}$ (3 m x $4 \mathrm{~m}$ ). Une fertilisation à l'Urée et au Diammonium Phosphate (DAP) avec la formule 120-60-60 $\left(\mathrm{N}, \mathrm{P}_{2} \mathrm{O}_{5}, \mathrm{~K}_{2} \mathrm{O}\right)$ a été utilisée. Le phosphore et le potassium ont été apportés en fond au repiquage et l'azote sous forme d'urée en couverture en 3 applications : Début Tallage (40\% de la dose), Initiation Paniculaire (40\% de la dose) et Montaison (20\% de la dose). Un traitement herbicide au Propanil à la dose de 8 1/ha a été utilisé pour le premier désherbage suivi de sarclages manuels en fonction $\mathrm{du}$ niveau d'infestation. Quatre variables ont été mesurés, dont le Cycle SemiMaturité (CSM), la Hauteur à maturité (H), le Nombre de panicules par $\mathrm{m}^{2}$ (PAN), et le Rendement en grains (RDT).

\section{Analyses statistiques}

Les essais ont été conduits en 2004 durant deux saisons successives, en Contre Saison Chaude (CSC) et Hivernage (HIV), dans deux sites différents, à Ndiaye dans le delta, et à Fanaye dans la moyenne vallée du Fleuve Sénégal.

L'analyse statistique a été faite avec l'aide du logiciel Plant Breeding Statistique (PLABSTAT) (UTZ, 1997), selon les modèles appropriés.

\section{RESULTATS}

Les valeurs moyennes par saison des variables étudiées sont résumées dans le Tableau 1.

En hivernage, dans le site de Fanaye, l'analyse de variance (ou test de Fisher) montre une différence très significative au seuil de $1 \%(\mathrm{P}<0,01)$ à significative au seuil de $5 \%(\mathrm{P}<0,05)$ entre les variétés et lignées pour les caractères cycle, rendement en grains et hauteur des plants, et non significatives pour le nombre de panicules par $\mathrm{m}^{2}$ (Tableau 1).

En contre saison chaude, les variétés présentent une différence très significative $(\mathrm{P}<0.01)$ pour l'ensemble des caractères. En plus, le cycle, la hauteur et le nombre de panicule par $\mathrm{m}^{2}$, ont des valeurs moyennes plus élevées en contre saison chaude qu'en hivernage.

En hivernage, dans le site de Ndiaye, les génotypes ont une différence très significative $(\mathrm{P}<0,01)$ pour les variables cycle, hauteur et nombre de panicules $/ \mathrm{m}^{2}$ (Tableau 2). En contre saison chaude, la différence entre les variétés et lignées est très significative $(\mathrm{P}<0,01)$ pour l'ensemble des variables. Comme pour le site de Fanaye, la valeur moyenne des caractères mesurés à Ndiaye est également plus élevée en contre saison chaude qu'en hivernage.

Les valeurs moyennes des variables mesurées combinées sur les deux sites sont résumées dans le Tableau 3. En hivernage comme en contre saison chaude, les variétés et lignées présentent des différences significatives $(\mathrm{P}<0,05)$ pour le nombre de panicules par $\mathrm{m}^{2}$, et très significatives $(\mathrm{P}<0,01)$ pour le cycle, le rendement en grains et la hauteur des plantes. Les valeurs moyennes des différents caractères mesurés sont également plus élevées en contre saison qu'en hivernage.

La valeur moyenne des variables, combinées sur les deux sites et les deux saisons, est résumée dans le Tableau 4. On note une différence très significative $(\mathrm{P}<0,01)$ pour l'ensemble des caractères étudiés.

Le Tableau 5 résume l'ensemble des valeurs de $\mathrm{F}$ pour la saison, le site, le génotype et leurs interactions respectives.

Les valeurs de F de l'analyse de variance montrent une différence très significative $\left(\mathrm{RH}_{0}\right.$ si $\left.\mathrm{F}_{\mathrm{cal}} \geq \mathrm{F}_{1-\alpha}\right)$, des génotypes pour l'ensemble des caractères étudiés. Un effet significatif de la Saison sur le Cycle et de l'interaction Génotypes x Site sur la Hauteur a également été noté. L'interaction Génotype $x$ Site a un effet faible sur le Cycle. L'effet combiné Génotype x Environnement x Saison, sur le Rendement est également faible. Il n'y a pas eu un effet Génotype x Environnement sur le Rendement.

Le Tableau 6 montre les rendements 
moyens des différentes lignées testées sur l'ensemble des sites et saisons combinés

La Figure 1 montre la répartition des variétés et lignées en fonction du cycle et du rendement. Les lignées se répartissent dans trois différents cycles (court, moyen et long). Les lignées les plus précoces se trouvent chez les hybrides intraspécifiques telles que WAS173-B-B-9-5, WAS196-B-4-3 et WAS173-B-B-6-4 avec 112,88, 113,25 et 113,38 JAS, respectivement (Figure 1). Les rendements moyens combinés sur sites et saisons varient entre 6,61 et 8,78 t.ha ${ }^{-1}$ (Figure 1, Tableau 6). Les meilleurs rendements ont été obtenus chez les cycles moyens et les cycles longs avec les lignées inraspécifiques WAS173-B-B-10-6-2 (8,78 t.ha $\left.{ }^{-1}\right)$, WAS183B-6-2-2 (8,74t.ha $\left.{ }^{-1}\right)$ et WAS173-B-B-4-2-4 $\left(8,44\right.$ t.ha $\left.{ }^{-1}\right)$, WAS173-B-B-10-6-5 (8,3 t.ha $\left.{ }^{-1}\right)$ et les lignées interspécifiques WAS191-7WAB-1-WAS-2 (8,4 t.ha $\left.{ }^{-1}\right)$, WAS187-2WAB-2-WAS-1 (8,15t.ha $\left.{ }^{-1}\right)$ et WAS126WAB-5-WAS-3-WAB-3-WAS-1 (7,74t.ha $\left.{ }^{-1}\right)$ (Tableau 6).

Il y a une corrélation positive $\left(\mathrm{r}=0,631^{* *}\right)$ entre le cycle et les autres caractères (Figure 1).

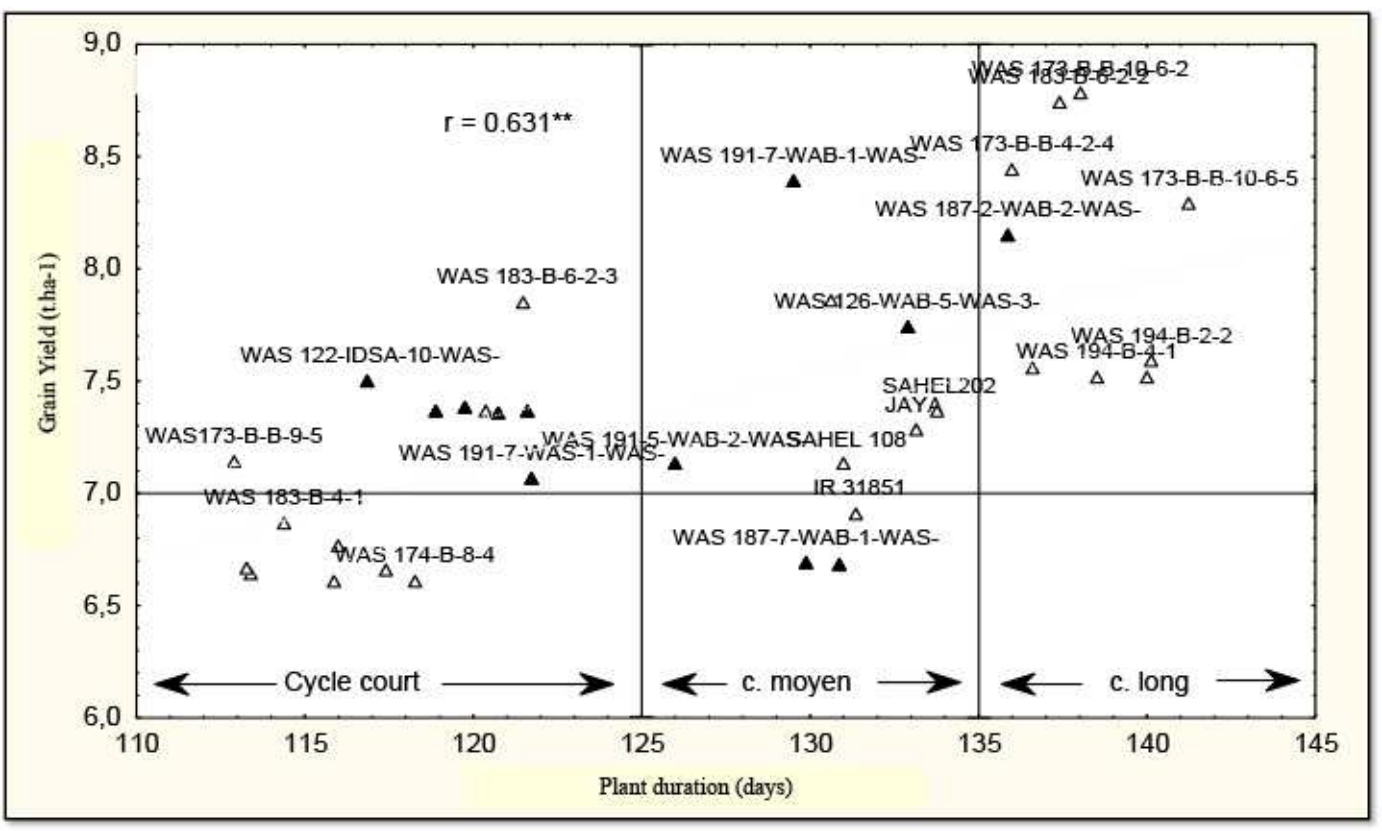

Figure 1 : Diversité des variétés et lignées en fonction du cycle et du rendement. ${ }^{1} \mathrm{P}$ désigne aussi "Pr( $>\mathrm{F}) "$ dont le seuil de rejet de $5 \%$ (si < 0,05), mais qui est identique à : $\mathrm{RH}_{0}$ si $\mathrm{F}_{\text {cal }} \geq \mathrm{F}_{1-\alpha}$.

Tableau 1 : Valeurs moyennes par saison des variables étudiées à Fanaye.

\begin{tabular}{lcc}
\hline \multicolumn{1}{c}{ Paramètres } & Hivernage & $\begin{array}{c}\text { Contre saison } \\
\text { Chaude }\end{array}$ \\
\hline Cycle (jours) & $120,81^{*}$ & $132,59 * *$ \\
Rendement $\left(\mathrm{t} . \mathrm{ha}^{-1}\right)$ & $7,41^{*} *$ & $7,34 * *$ \\
Hauteur $(\mathrm{cm})$ & $86,46 * *$ & $91,53 * *$ \\
Panicules $/ \mathrm{m}^{2}$ & $374,87 \mathrm{~ns}$ & $437,96 * *$ \\
\hline$* * *$ différence statistiquement significatif à $\mathrm{P}=0,05, \mathrm{P}=0,01$, ANOVA F-test.
\end{tabular}


Tableau 2 : Valeurs moyennes par saison des variables étudiées à Ndiaye.

\begin{tabular}{lcc}
\hline Paramètres & HIV & CSC \\
\hline Cycle (jours) & $115 * *$ & $138,53 * *$ \\
Rendement $\left(\mathrm{t}^{*} \mathrm{ha}^{-1}\right)$ & 6,76 & $8,09 * *$ \\
Hauteur $(\mathrm{cm})$ & $102,01 * *$ & $105,39 * *$ \\
Panicules $/ \mathrm{m}^{2}$ & $346,19 * *$ & $405,61 *$ \\
\hline$* *, *$ différence statistiquement significatif à $\mathrm{P}=0,05, \mathrm{P}=0,01$, \\
ANOVA F-test (à 5\%, 1\% et 1 pour 1 000);
\end{tabular}

Tableau 3: Valeurs moyennes des variables étudiées par saison combinée sur les deux sites.

\begin{tabular}{lcc}
\hline Paramètres & Hivernage & CSC \\
\hline Cycle (jours) & $117,91 * *$ & $135,56 * *$ \\
Rendement $\left(\mathrm{t}^{*} \mathrm{ha}^{-1}\right)$ & $7,08 * *$ & $7,72 * *$ \\
Hauteur $(\mathrm{cm})$ & $94,24 * *$ & $98,46 * *$ \\
Panicules $/ \mathrm{m}^{2}$ & $360,53 *$ & $421,79 * *$ \\
\hline$* * *$ : différence statistiquement significatif à $\mathrm{P}=0,05, \mathrm{P}=0,01$, \\
ANOVA F-test.
\end{tabular}

Tableau 4: Valeurs moyennes des variables étudiées combinées sur les deux sites et les deux saisons.

\begin{tabular}{lc}
\hline Paramètres & Moyenne \\
\hline Cycle (jours) & $126,73 * *$ \\
Rendement $\left(\mathrm{t} . h a^{-1}\right)$ & $7,40 * *$ \\
Hauteur $(\mathrm{cm})$ & $96,35 * *$ \\
Panicules $/ \mathrm{m}^{2}$ & $391,16 * *$ \\
\hline$* *, *$ différence statistiquement significatif à $\mathrm{P}=0,05$, \\
$\mathrm{P}=0,01$, ANOVA F-test.
\end{tabular}

Tableau 5: Valeurs de F de l'analyse de variance du génotype (G), du site (E), de la saison (S), et leurs interactions pour les différents caractères.

\begin{tabular}{|c|c|c|c|c|c|c|}
\hline & $\mathbf{S}$ & $\mathbf{E}$ & $\mathbf{G}$ & SG & $E \times G$ & SEG \\
\hline Cycle (jours) & $2,34 *$ & 1,28 & $15,58 * *$ & 0,34 & $1,43+$ & 0,68 \\
\hline Rendement (t.ha $\left.{ }^{-1}\right)$ & 0,94 & 1,25 & $2,32 * *$ & 0,82 & 0,61 & $1,42+$ \\
\hline Hauteur $(\mathrm{cm})$ & 1,08 & 1,01 & $10,64 * *$ & 1,07 & $1,79 *$ & 0,91 \\
\hline Panicules par $\mathrm{m}^{2}$ & 0,78 & 0,67 & $4,32 * *$ & 0,49 & 0,81 & 0,68 \\
\hline
\end{tabular}


T. GUEYE et al. / Int. J. Biol. Chem. Sci. 10(1): 232-241, 2016

Tableau 6: Rendements moyens combinées sur sites et saisons des lignées.

\begin{tabular}{|c|c|c|c|c|c|}
\hline $\mathbf{N}^{\circ}$ & Génotypes & Cycle (JAS) & Hauteur (cm) & Panicules $/ \mathrm{m}^{2}$ & $\begin{array}{c}\text { Rendements } \pm \\
\text { Ecart type }\left(\text { t.ha }^{-1}\right)\end{array}$ \\
\hline 1 & WAS 173-B-B-10-6-2 & 138 & 112,25 & 415 & $8,78 \pm 1,19$ \\
\hline 2 & WAS 183-B-6-2-2 & 138 & 94,63 & 405 & $8,74 \pm 0,71$ \\
\hline 3 & WAS 173-B-B-4-2-4 & 136 & 95,5 & 459 & $8,44 \pm 0,95$ \\
\hline 4 & WAS 191-7-WAB-1-WAS-2 & 130 & 89 & 416 & $8,39 \pm 0,91$ \\
\hline 5 & WAS173-B-B-10-6-5 & 142 & 115,88 & 395 & $8,29 \pm 0,99$ \\
\hline 6 & WAS187-2-WAB-2-WAS-1 & 1368 & 99,13 & 453 & $8,15 \pm 1,11$ \\
\hline 7 & WAS196-B5-2 & 131 & 98,75 & 422 & $7,86 \pm 0,87$ \\
\hline 8 & WAS183-B-6-2-3 & 122 & 90,63 & 369 & $7,85 \pm 1,05$ \\
\hline 9 & WAS126-WAB-5-WAS-3-WAB-3-WAS-1 & 133 & 90,38 & 437 & $7,74 \pm 0,93$ \\
\hline 10 & WAS194-B-2-2 & 140 & 107,5 & 410 & $7,59 \pm 0,72$ \\
\hline 11 & WAS196-B-5-1 & 137 & 88,75 & 397 & $7,56 \pm 1,06$ \\
\hline 12 & WAS194-B-4-1 & 139 & 113,25 & 372 & $7,52 \pm 0,51$ \\
\hline 13 & WAS194-B-2-1 & 140 & 113 & 389 & $7,52 \pm 0,69$ \\
\hline 14 & WAS122-IDSA-10-WAS-10-WAB-2-WAS-1 & 117 & 80,75 & 377 & $7,50 \pm 1,46$ \\
\hline 15 & WAS127-IDSA-10-WAS-5-WAB-1-WAS-2 & 120 & 88,25 & 382 & $7,38 \pm 0,45$ \\
\hline 16 & WAS122-IDSA-1-WAS-3-WAB-1-WAS-1 & 119 & 85,88 & 367 & $7,37 \pm 0,66$ \\
\hline 17 & WAS191-5-WAB-1-WAS-3 & 122 & 90,13 & 386 & $7,37 \pm 0,29$ \\
\hline 18 & WAS173-B-B-2-1-5 & 121 & 105,63 & 366 & $7,37 \pm 0,91$ \\
\hline 19 & WAS191-4-WAB-1-WAS-1 & 121 & 90,25 & 377 & $7,36 \pm 0,86$ \\
\hline 20 & WAS173-B-B-9-5 & 113 & 98 & 368 & $7,14 \pm 0,99$ \\
\hline 21 & WAS191-5-WAB-2-WAS-2 & 126 & 80,75 & 372 & $7,13 \pm 0,78$ \\
\hline 22 & WAS191-7-WAS-1-WAS-1 & 122 & 84 & 372 & $7,07 \pm 0,91$ \\
\hline 23 & WAS183-B-4-1 & 115 & 101,5 & 394 & $6,87 \pm 0,30$ \\
\hline 24 & WAS175-B-21-4 & 116 & 104,75 & 363 & $6,77 \pm 1,12$ \\
\hline
\end{tabular}


T. GUEYE et al. / Int. J. Biol. Chem. Sci. 10(1): 232-241, 2016

\begin{tabular}{llcccc}
\hline 25 & WAS187-7-WAB-1-WAS-1 & 130 & 80 & 445 & $6,69 \pm 0,75$ \\
26 & WAS187-2-WAB-1-WAS-1 & 131 & 94 & 455 & $6,68 \pm 0,59$ \\
27 & WAS196-B-4-3 & 113 & 94,88 & 350 & $6,67 \pm 0,89$ \\
28 & WAS173-B-B-5-3 & 118 & 105,5 & 305 & $6,66 \pm 0,89$ \\
29 & WAS173-B-B-6-4 & 114 & 102 & 340 & $6,64 \pm 0,83$ \\
30 & WAS173-B-B-4-2 & 116 & 109 & 301 & $6,61 \pm 0,65$ \\
31 & WAS174-B-8-4 & 119 & 100,5 & 320 & $6,61 \pm 0,84$ \\
& Témoins & & & \\
32 & Sahel 202 & 134 & 97,5 & 415 & $7,37 \pm 1,35$ \\
33 & JAYA & 133 & 100,25 & 436 & $7,28 \pm 1,02$ \\
34 & Sahel 108 & 131 & 86,75 & 439 & $6,13 \pm 0,97$ \\
35 & IR 31851 & 132 & 83,25 & 434 & 0,75 \\
& PPDS5 & 6,53 & 8,6 & 54,8 & \\
\hline
\end{tabular}




\section{DISCUSSION}

A l'exception du nombre de panicules par $\mathrm{m}^{2}$ mesuré à Fanaye, en hivernage (Tableau 1), l'analyse de variance sur chaque site et/ou saison, combiné ou non, a révélé un effet significatif du génotype sur l'ensemble des caractères étudiés. Cette variation génotypique est un facteur important pour l'amélioration de caractères (Okeno, 2001; Pliura et al., 2014). Il y a également un effet de la saison sur le cycle et une interaction $\mathrm{G} x$ E sur la hauteur. Il n'y a pas eu un effet G x E sur le rendement et le nombre de panicules par m2 (Tableau 4). L'effet de la saison sur le cycle montre l'influence des variations saisonnières sur la durée du cycle. Ce résultat est en conformité avec de nombreuses études sur différentes espèces cultivées ( $\mathrm{Wu}$ et al., 2013 ; Zhao et al., 2015). Durant la contre saison chaude, les basses températures en période végétative ont des effets sur l'allongement du cycle. Afin de réduire les pertes liées à la baisse de température, Chen et al. (2013) ont modélisé à l'aide des données météorologiques et de télédétection les risques de dégâts par le froid. Dans un système à double culture, l'âge des semis est aussi un facteur important à prendre en compte car lorsqu'elle est prolongé à $30-35$ jours, cela à une incidence négative sur les rendements en grains (Hu et al., 2015). L'interaction significative $G \times$ E notée sur la hauteur indique que la variation des sites a une influence sur la taille des lignées. L'absence d'effets Génotype x Saison ( $G \times x$ S) sur l'ensemble des caractères mesurés, montre que la variation saisonnière ne semble pas influer sur le développement des lignées. L'absence d'interaction $G \times x$ sur le rendement constitue un résultat intéressant, qui renseigne sur le potentiel des lignées notamment par rapport à leur stabilité c'est à dire leur aptitude à donner de bonnes performances sur plusieurs sites. Ce résultat est en cohérence avec les études sur les variétés modernes caractérisées par une interaction $\mathrm{G} \times \mathrm{E}$ de plus en plus réduite et une stabilité de plus en plus large (Vitaa et al., 2010). Elfadil et al. (2014), en travaillant sur le mil au Soudan, ont mis en relief l'importance de la sélection de variétés performantes et stables pour impulser les programmes et identifier des génotypes intéressants en terme de stabilité de rendement décomposé en deux composants dont une stabilité statique et une stabilité dynamique. Les performances des variétés et lignées dans les différents sites et saisons, combinés ou non, permettent de distinguer trois catégories de variétés et lignées. Parmi ces trois groupes, les variétés et lignées performantes sur plusieurs sites et saisons semblent être les plus intéressantes. C'est un matériel de choix qui présente une plus grande adaptation. Pour une meilleure productivité en riziculture irriguée, des rendements élevés et stables combinés avec des cycles prévisibles dans des conditions environnementales variées en terme de températures et de photopériode, sont en effet, des facteurs essentiels. Les variétés et lignées qui sont plus performantes sur un site ou une saison ne sont cependant pas à négliger. Il y a une diversité des lignées en fonction du cycle et du rendement avec une corrélation positive $r=0,631^{* *}$. Les meilleurs rendements combinés sur sites et saisons ont été enregistrés chez les lignées à cycle moyen dont la WAS173-B-B-10-6-2 et la WAS183B-6-2-2. Selon Yuan et al. (2011), les meilleurs rendements sont obtenus davantage sur des lignées testées dans un dispositif agronomique, que sur des lignées choisies selon leurs caractères agronomiques. Ces orientations de Yuan et al. (2011) sont en cohérence avec les résultats obtenus et constituent une bonne source d'inspiration à prendre davantage en compte dans le processus de vulgarisation de nouvelles variétés.

\section{Conclusion}

L'étude du comportement des lignées intra- et interspécifiques a mis en évidence une diversité du matériel génétique. Les lignées sont réparties en trois cycles (court, moyen et long) avec une corrélation positive entre le cycle et le rendement. Les variétés et lignées performantes sur plusieurs sites et 
saisons, ont une plus grande aptitude à double culture. Elles devraient ainsi contribuer à l'amélioration de la production dans la vallée du Fleuve Sénégal.

\section{CONFLIT D'INTERÊT}

Les auteurs déclarent qu'il n'y a pas de conflits d'intérêts.

\section{CONTRIBUTIONS DES AUTEURS}

TG est l'investigateur principal ; SN et $\mathrm{AD}$ ont apporté leur contribution dans la révision et l'amélioration du manuscrit.

\section{REFERENCES}

Banito A, Kadai EA, Anasanya A, Séré Y. 2012. Evaluation of rice genotype to bacterial leaf blight in Togo. Int. J. Biol. Chem. Sci., 6(4):1548-1556.

Bimpong IK, Manneh B, Sock M, Diaw F, Amoah NKA, Ismail AM, Gregorio G, Singh RK, Wopereis M. 2016. Improving salt tolerance of lowland rice cultivar 'Rassi' through marker-aided backcross breeding in West Africa. Plant Science, 242: 288-299.

Bustos DV, AK Hasan, Reynolds MP, Calderini DF. 2013. Combining high grain number and weight through a $\mathrm{DH}$ population to improve grain yield potential of wheat in high-yielding environments. Field Crops Research, 145: 106-115.

Cheng Y, Huang J, Han Z, Guo J, Zhao Y, Wang X, Guo R. 2013. Cold Damage Risk Assessment of Double Cropping Rice in Hunan, China. Journal of Integrative Agriculture, 12(2): 352-363.

Elfadil MAB, Abdelbagi MA, Adam MA, Ismael MI, Parzies HK, Haussmann BIG. 2014. Patterns of pearl millet genotypeby-environment interaction for yield performance and grain iron (Fe) and zinc (Zn) concentrations in Sudan. Field Crops Research, 166: 82-91.

Gaouna BO, Traoré ER, Assane S, Zongo JD. 2012. Caractérisation agromorphologique des accessions de riz adventices (Oryzasp) collectés dans les riziers de la zone interfluve du Tchad. Int. J. Biol. Chem. Sci., 5(6): 1774-1791.

Goalbaye T, Guisse A, Ndiaye M, Tissou M. 2013. Les populations de maïs améliorées et adaptées à la sécheresse pour les zones à faible pluviométrie au Tchad. Int. J. Biol. Chem. Sci., 7(6): 2275-2282.

Haefele SM, Johnson DE, MBodj D, Wopereis MCS, Miezan KM. 2004. Field screening of diverse rice genotypes for weed competitiveness in irrigated lowland ecosystems. Field Crops Research, 88(1) : 39-56.

Hu Z, Liu Y, Huang L, Peng S, Nie L, Cui K, Huang L, Wang F. 2015. Premature heading and yield losses caused by prolonged seedling age in double cropping rice. Field Crops Research, 183: 147-155.

Jones PM, Dingkuhn M, Aluko GK, Semon M. 1997. Interspecific Oryza sativa L. x O. glaberrima Steudt. 1997. Progenies in upland rice improvement. Euphytica, 92: 327-246.

Li C, Tao Z, Liu P, Zhang J, Zhuang K, Dong S, Zhao M. 2015. Increased grain yield with improved photosynthetic characters in modern maize parental lines. Journal of Integrative Agriculture, 14(9): 17351744.

Liang S, Ren G, Liu J, Zhao X, Zhou M, McNeil D, Ye G. 2014. Genotype-byenvironment interaction is important for grain yield in irrigated lowland rice. Field Crops Research, 180: 90-99.

Ndjiondjop MN, Manneh B, Cissoko M, Drame NK, Kakai RG, Bocco R, Baimey H, Wopereis M. 2010. Drought resistance in an interspecific backcross population of rice (Oryza spp.) derived from the cross WAB56-104 (O. sativa) × CG14 (O. glaberrima). Plant Science, 179(4): 364373.

Okeno JA. 2001. Genotypic variation in morphological traits of barley as affected by nitrogen supply. In Plant Nutrition Food Security and Sustainability of AgroEcosystems, Horst WJ (Eds). Kluwer Academic Publishers; 64-65. 
Pliura A, Suchockas V, Sarsekova D, Gudynaite V. 2014. Genotypic variation and heritability of growth and adaptive traits, and adaptation of young poplar hybrids at northern margins of natural distribution of Populus nigra in Europe. Biomass and Bioenergy, 70: 513 - 529.

UTZ HF. 1997. Ein Computerprogramm zur Statistischen Analyse von Pflanzenzuechterischen Experiment. Version 2B. Institut fuer Pflanzenzuechtung, Saatgutforschung und Populationsgenetik, Universitaet Hohenheim.

Vitaa PD, Mastrangeloa AM, Matteua L, Mazzucotellib E, Virzì N, Palumboc M, Stortod ML, Rizzab F, Cattivelli L. 2010. Genetic improvement effects on yield stability in durum wheat genotypes grown in Italy. Field Crops Research, 119: 6877.

Wu W, Nie L, Liao Y, Shah F, Cui K, Wang Q, Lian Y, Huang J. 2013. Toward yield improvement of early-season rice: Other options under double rice-cropping system in central China. European Journal of Agronomy, 45: 75-86.

Yacouba AS, Moussa OZ, Kadri A, Kaka S, Ibroh G. 2013. Participatory varietal selection: an approach used for the selection of lowland NERICA varieties in Niger. Int. J. Biol. Chem. Sci., 7(6): 22022013.

Yuan W, Peng S, Cao C, Virk P, Xing D, Zhang Y, Visperas RM, Laza RC. 2011. Agronomic performance of rice breeding lines selected based on plant traits or grain yield. Field Crops Research, 121: 168-174.

Zhao J, Yang X, Dai S, Lv S, Wang J. 2015. Increased utilization of lengthening growing season and warming temperatures by adjusting sowing dates and cultivar selection for spring maize in Northeast China. European Journal of Agronomy, 67: 12-19. 